\title{
Vibrational deactivation on chemically reactive potential surfaces: An exact quantum study of a low barrier collinear model of H + FH, D + FD, H + FD, and $\mathbf{D}+\mathbf{F H}^{\mathbf{a})}$
}

\author{
George C. Schatz ${ }^{\text {b) }}$ and Aron Kuppermann \\ Arthur Amos Noyes Laboratory of Chemical Physics, ${ }^{\text {c) }}$ California Institute of Technology, Pasadena, \\ California 91125 \\ (Received 23 March 1979; accepted 12 June 1979)
}

\begin{abstract}
We study vibrational deactivation processes on chemically reactive potential energy surfaces by examining accurate quantum mechanical transition probabilities and rate constants for the collinear $\mathbf{H}+\mathbf{F H}(v)$, D $+\mathrm{FD}(v), \mathrm{H}+\mathrm{FD}(v)$, and $\mathrm{D}+\mathrm{FH}(v)$ reactions. A low barrier $(1.7 \mathrm{kcal} / \mathrm{mole})$ potential surface is used in these calculations, and we find that for all four reactions, the reactive inelastic rate constants are larger than the nonreactive ones for the same initial and final vibrational states. However, the ratios of these reactive and nonreactive rate constants depend strongly on the vibrational quantum number $v$ and the isotopic composition of the reagents. Nonreactive and reactive transition probabilities for multiquantum jump transitions are generally comparable to those for single quantum transitions. This vibrationally nonadiabatic behavior is a direct consequence of the severe distortion of the diatomic that occurs in a collision on a low barrier reactive surface, and can make chemically reactive atoms like $\mathrm{H}$ or D more efficient deactivators of HF or DF than nonreactive collision partners. Many conclusions are in at least qualitative agreement with those of Wilkin's three dimensional quasiclassical trajectory study on the same systems using a similar surface. We also present results for $\mathbf{H}+\mathbf{H F}(v)$ collisions which show that for a higher barrier potential surface (33 rather than $1.7 \mathrm{kcal} / \mathrm{mole}$ ), the deactivation process becomes similar in character to that for nonreactive partners, with $v \rightarrow v-1$ processes dominating.
\end{abstract}

\section{INTRODUCTION}

The collisional deactivation of vibrationally excited diatomics like HF is an important rate process in determining the efficiency of many chemical lasers. While vibrational deactivation by nonreactive collision partners such as rare gases has been well studied ${ }^{1}$ and is gener ally well understood at least qualitatively, the deactivation of diatomics by atoms and molecules with which chemical reaction is also possible (i. e., on a chemically reactive potential energy surface) has rarely been studied, in part because of the difficulty associated with accurate quantum calculations for reactive collision systems. Such deactivation processes can, however, play an important role in chemical lasers, since there are often many species present in such systems which are capable of reacting with the vibrationally excited molecules responsible for lasing. In the $\mathrm{HF}$ lasers, for example, the vibrationally excited HF can react with (or be deactivated by) the $\mathrm{H}$ and $\mathrm{F}$ atoms which are present as reagents and products in the primary pumping reaction $\mathrm{F}+\mathrm{H}_{2}-\mathrm{HF}+\mathrm{H}^{2-4}$

In this paper, we examine vibrational deactivation processes on chemically reactive surfaces in more detail in an attempt to understand both qualitatively and quantitatively such dynamical features as the importance of multiquantum jump transitions in vibrational deactivation, the importance of reactive versus nonre-

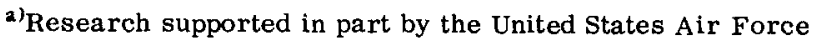
Office of Scientific Research.

b 'Work performed in partial fulfillment of the requirements for the Ph. D. in Chemistry at the California Institute of Technology. Present address: Department of Chemistry, Northwestern University, Evanston, Ill. 60201.

c) Contribution No. 5936 .
}

active pathways to deactivation (when applicable) and the effect of isotopic substitution on deactivation rates. In all applications, accurate quantum mechanical methods $^{5-10}$ will be used to treat the collision dynamics, but the collisions will be constrained to be collinear. The accurate quantum treatment enables us to assess the features of vibrational deactivation without fear that this assessment may be biased by approximations in the dynamics. The collinear approximation makes the quantum treatment tractable while still providing a realistic dynamical description. ${ }^{8,9}$

We apply these calculations to the deactivation of $\mathrm{HF}$ by $\mathrm{H}$ atoms, (and isotopic counterparts), modelling the surface as having a low barrier of $1.7 \mathrm{kcal} / \mathrm{mole}$. This deactivation process has been the subject of several experimental $^{2-4}$ and theoretical $1^{10,11,12}$ studies, few of which are in good agreement with each other. For example, three experimental determinations of the $\mathrm{H}+\mathrm{FH}(v=1)$ deactivation rate give rate constants at $300^{\circ} \mathrm{K}$ of $<(7 \pm 4)$ $\times 10^{11},{ }^{3} \leq 9 \times 10^{9},{ }^{2(a)}$ and $(1.4 \pm 0.4) \times 10^{11} \mathrm{~cm}^{3} / \mathrm{mole} \mathrm{sec},{ }^{4}$ while two classical trajectory calculations of this same quantity give $2.2 \times 10^{1211}$ and $\sim 1 \times 10^{1212} \mathrm{~cm}^{3} / \mathrm{mole}$ sec. Experimental and theoretical comparisons of the rate constants for the isotopically related deactivation processes $\mathrm{D}+\mathrm{FD}(v=1), \mathrm{D}+\mathrm{FH}(v=1)$, and $\mathrm{H}+\mathrm{FD}(v=1)$ are also poor. ${ }^{4}$ The theoretical studies have used LEPS type surfaces which were optimized for only $\mathrm{F}+\mathrm{H}_{2}$ configurations, then applied to $\mathrm{H}+\mathrm{FH}$ collisions. While this procedure has been successful for deactivation of $\mathrm{HF}$ by $\mathrm{F}^{2(\mathrm{~b}), 13,14}$ (but see Ref. 15), Bender et al. and others ${ }^{16}$ have shown that the low (1-2 kcal/mole) barrier obtained from the LEPS surfaces differs very substantially from the analogous $a b$ initio barrier ( $49 \mathrm{kcal} / \mathrm{mole}$ ) and, therefore, could be seriously in error. Recent experimental evidence ${ }^{17}$ corroborates this high barrier 
prediction. Our results using a low barrier surface might, then, not be realistic for $\mathrm{H}+\mathrm{FH}$, although they should be representative of deactivation in other reactions having surfaces with low barriers. To provide some indication of the effect of a higher barrier, we also present results for deactivation of $\mathrm{HF}$ in the linear $\mathrm{HHF}$ geometry, where the barrier is $33 \mathrm{kcal} / \mathrm{mole}$. A more extensive study of $\mathrm{H}+\mathrm{FH}$ deactivation processes on surfaces of varying barrier heights will be presented in a future publication. ${ }^{18}$

The specific systems we will investigate are

$$
\begin{aligned}
\mathrm{H}+\mathrm{FH}(v=0-3) & -\mathrm{HF}\left(v^{\prime}=0-2\right)+\mathrm{H} \\
& -\mathrm{H}+\mathrm{FH}\left(v^{\prime}=0-2\right) \\
\mathrm{D}+\mathrm{FD}(v=0,1) & -\mathrm{DF}\left(v^{\prime}=0\right)+\mathrm{D} \\
& -\mathrm{D}+\mathrm{FD}\left(v^{\prime}=0\right) \\
\mathrm{D}+\mathrm{FH}(v=0,1) & -\mathrm{DF}\left(v^{\prime}=0\right)+\mathrm{H} \\
& -\mathrm{D}+\mathrm{FH}\left(v^{\prime}=0\right) \\
\mathrm{H}+\mathrm{FD}(v=0,1) & -\mathrm{HF}\left(v^{\prime}=0\right)+\mathrm{D} \\
& \rightarrow \mathrm{H}+\mathrm{FD}\left(v^{\prime}=0\right)
\end{aligned}
$$

Reactions (R. 3) and (R.4) are actually considered in the same calculation, since transitions between all possible open states of both reagents and products are determined simultaneously. In all the above reactions, the linear collision complex has the $\mathrm{F}$ atom between the hydrogens or deuteriums. The actual three dimensional collision includes other configurations, such as

$$
\mathrm{H}+\mathrm{HF}(v=1)-\mathrm{H}+\mathrm{HF}\left(v^{\prime}=0\right)
$$

(where the $\mathrm{F}$ atom is on one end the 3 atom system), which we will also consider. Just which geometry will be more representative of the real world will depend on how the barrier height varies with orientation. We will show that for the surface considered, (R.1) appears to be more representative than (R.5). Note that all atoms in the collision are assumed distinguishable in the collinear model. This allows us to separate the reactive and nonreactive deactivation processes, and is analogous to the distinction made in the classical treatments. ${ }^{6,7}$ Of course, the correct three dimensional treatment of $\mathrm{H}+\mathrm{FH}$ might lead to quantum interference oscillations in cross sections. Such oscillations have, in fact, been observed in quantum calculations on $\mathrm{H}+\mathrm{H}_{2},{ }^{14}$ where their effect on thermal rate constants is apparently small.

To summarize the rest of this paper, in Sec. II, we briefly describe details of the calculation, while in Sec. III, transition probabilities and rate constants for system (R. 1-R.5) are presented and analyzed. A summary of conclusions is presented in Sec. IV.

\section{THE CALCULATION}

A coupled channel propagation technique ${ }^{5}$ was used to solve the Schrödinger equation for the collinear reactive and nonreactive collisions. This method has been previously applied to $\mathrm{H}+\mathrm{H}_{2},{ }^{19} \mathrm{~F}+\mathrm{H}_{2},{ }^{6,20}$ and $\mathrm{F}+\mathrm{D}_{2}{ }^{7}$ and is more thoroughly described in Ref. 6. Between 10 and 12 channels were included in the vibrational basis sets with
1 to 4 open and the rest closed. Convergence of the transition probabilities with respect to the addition of closed channels was tested, and this, together with tests of flux conservation and microscopic reversibility indicate that the results presented are accurate to $1 \%$ or better. The potential surface used was Muckerman's surface $5,{ }^{21,22}$ the LEPS parameters of which are given in Ref. 6. ${ }^{23}$ Figure 1 depicts equipotential contours of this surface. The potential has a small barrier in the region of the cross in the figure, with a small hollow in the top of this barrier. The saddle points on both sides of this hollow have a potential of $1.75 \mathrm{kcal} / \mathrm{mole}(0.076$ $\mathrm{eV}$ ) relative to separated $\mathrm{HF}+\mathrm{H}$, and the hollow itself is $0.5 \mathrm{kcal}$ deep relative to these saddle points. The vibrational energy levels (including zero point energy) of $\mathrm{HF}$ are $5.8,17.1,27.8$, and $38.1 \mathrm{kcal} / \mathrm{mole}$ for $v=0,1,2$, and 3 , respectively. For DF, they are 4.2 , $12.5,20.5$, and $28.1 \mathrm{kcal} / \mathrm{mole}$ for these same levels.

The primary results of the calculation are the transition probabilities (which are analogous to the $3 \mathrm{D}$ cross sections) and the one dimensional thermal rate constants. These results cannot be directly compared with those of $3 \mathrm{D}$ calculations or with experiment without additional assumptions such as the assignment of an impact parameter dependence to the reaction probability. ${ }^{8}$ Alternatively, ratios of rate constants can be compared. This is a dangerous procedure if no allowance for rotational states is made, but often gives qualitatively useful comparisons as will be apparent in Sec. III B.

\section{RESULTS}

\section{A. Transition probabilities}

We denote the transition probability from vibrational state $v$ of the reagent to state $v^{\prime}$ of the product by the

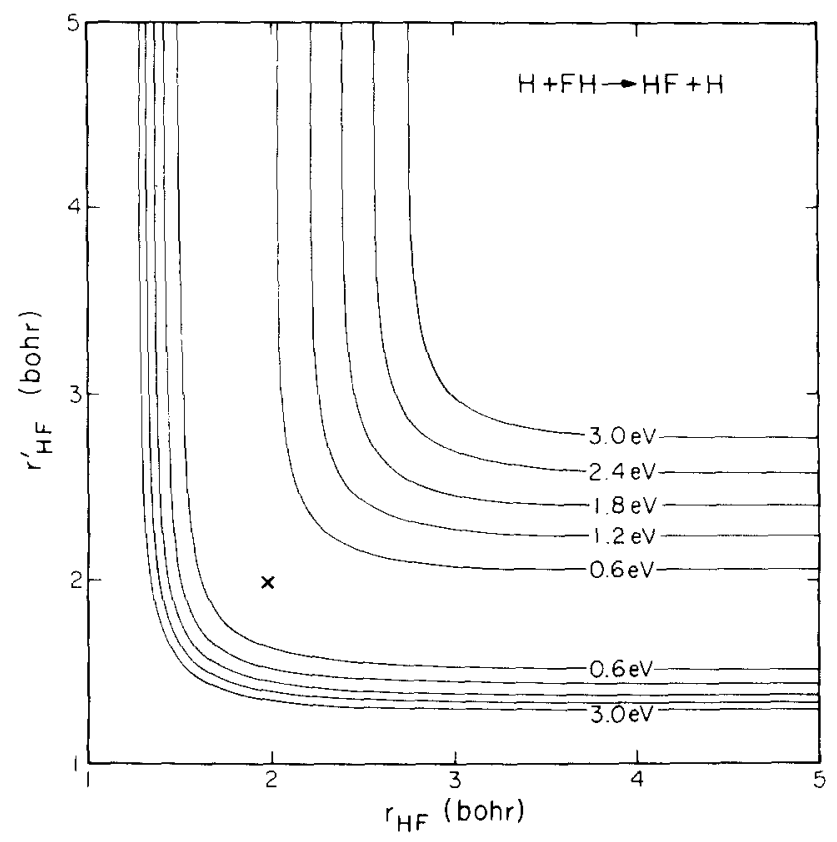

FIG. 1. Equipotential contours for the collinear $\mathrm{H}+\mathrm{FH}$ surface as a function of the two HF diatomic internuclear distances $r_{\mathrm{HF}}$ and $r_{\mathrm{HP}}^{\prime}$ 


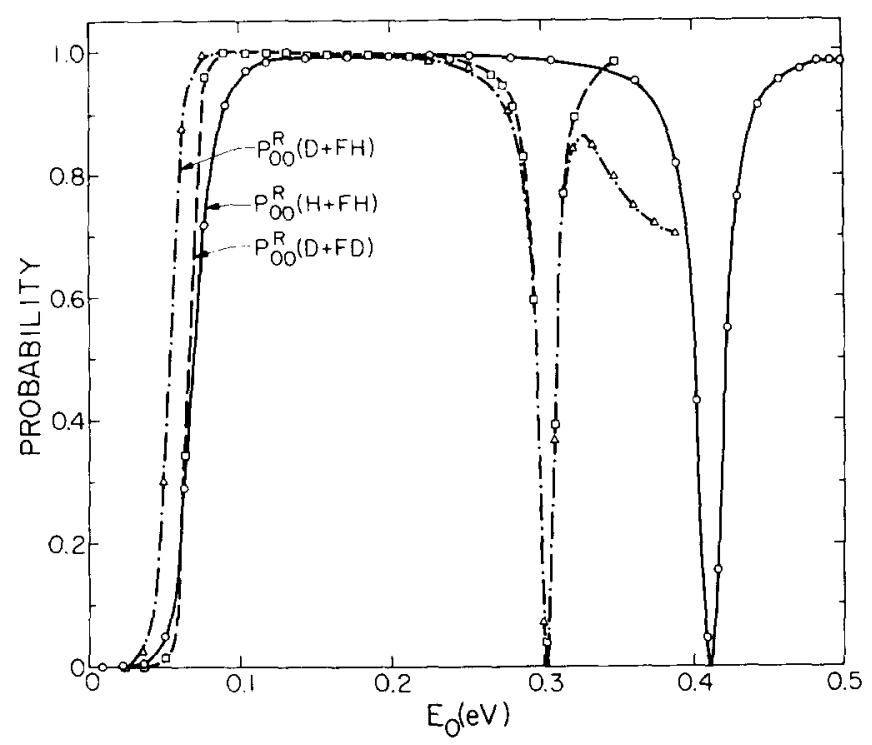

FIG. 2. Reaction probability $P_{00}^{R}$ for $\mathrm{H}+\mathrm{FH}$ (solid curve and circles), D+FD (dashed curve and squares), and D+FH (dash-dotted curve and triangles) as a function of the reagent relative translational energy $E_{0}$ appropriate for each collision system.

symbol $P_{v v^{\prime}}$. If the collision is reactive, we use the superscript $R$ (i.e., $P_{v v^{\prime}}^{R}$ ) and if nonreactive, the superscript $V\left(P_{v v^{\circ}}^{V}\right)$. To identify the different reactions (R. 1-R.5) above, we specify the reagents in parenthesis after the transition probability. Thus $P_{10}^{R}(\mathrm{H}+\mathrm{FD})$ means the reaction probability for $\mathrm{H}+\mathrm{FD}(v=1)-\mathrm{HF}\left(v^{\prime}\right.$ $=0)+\mathrm{D}$ while $P_{10}^{V}(\mathrm{H}+\mathrm{FD})$ implies the nonreactive probability for $\mathrm{H}+\mathrm{FD}(v=1) \rightarrow \mathrm{H}+\mathrm{FD}\left(v^{\prime}=0\right)$.

We first consider the ground vibrational state reaction probabilities $P_{00}^{R}(\mathrm{H}+\mathrm{FH}), P_{00}^{R}(\mathrm{D}+\mathrm{FD})$ and $P_{00}^{R}(\mathrm{D}+\mathrm{FH})$. These are plotted in Fig. 2 as a function of the reagent relative translational energy $E_{0}$. (More generally, we let $E_{v}$ be the translational energy relative to vibrational state $v$ of the reagent diatomic molecule.) $P_{00}^{R}(\mathrm{H}+\mathrm{FD})$ can be obtained from $P_{00}^{R}(\mathrm{D}+\mathrm{FH})^{24}$ by shifting the latter curve in Fig. 2 upwards in energy by $0.069 \mathrm{eV}$ (which is the difference between the HF and DF zero point energies). The $P_{00}^{R}$ curves are quite similar in shape to the analogous reaction probability $P_{00}^{R}\left(\mathrm{H}+\mathrm{H}_{2}\right)$ which has been analyzed previously in detail. ${ }^{25,26}$ At low energies $\left(E_{0}\right.$ $<0.03 \mathrm{eV}$ ) the reaction probabilities are small. This is the expected behavior when barrier tunnelling (in an adiabatic sense) is occurring. The $P_{00}^{R}$ curves then show a sudden rise to nearly unit probability. The energies at which $P_{00}^{R}$ equals 0.01 are $E_{0}=0.04 \mathrm{eV}$ for $\mathrm{H}+\mathrm{FH}, 0.050$ $\mathrm{eV}$ for $\mathrm{D}+\mathrm{FD}, 0.030 \mathrm{eV}$ for $\mathrm{D}+\mathrm{FH}$, and $0.099 \mathrm{eV}$ for $\mathrm{H}+\mathrm{FD}$. The ordering of these effective threshold energies (D+FH lowest, then $\mathrm{H}+\mathrm{FH}, \mathrm{D}+\mathrm{FD}$, and $\mathrm{H}+\mathrm{FD})$ is completely analogous to the ordering previously found for the series $\mathrm{D}+\mathrm{H}_{2}, \mathrm{H}+\mathrm{H}_{2}, \mathrm{D}+\mathrm{D}_{2}$, and $\mathrm{H}+\mathrm{D}_{2}{ }^{27}$ and may be explained in terms of the vibrationally adiabatic barriers. The $\mathrm{H}+\mathrm{HF}$ threshold energy is identical to that obtained by $\mathrm{Baer}^{10}$ in his quantum coplanar study of this same reaction (on the Wilkins potential surface). ${ }^{11}$ At higher energies, the $P_{00}^{R}$ curves show sudden dips due to internal excitation resonances. ${ }^{19}$ These resonances are at $0.412 \mathrm{eV}$ for $\mathrm{H}+\mathrm{FH}, 0.302 \mathrm{eV}$ for $\mathrm{D}+\mathrm{FD}$ and $\mathrm{D}+\mathrm{FH}$ and $0.371 \mathrm{eV}$ for $\mathrm{H}+\mathrm{FD}$. We shall present a more complete analysis of the $\mathrm{H}+\mathrm{FH}$ resonance in a separate paper. ${ }^{28}$

The vibrationally inelastic transition probabilities $P_{10}^{R}$ and $P_{10}^{V}$ for $\mathrm{H}+\mathrm{FH}$ are presented in Fig. $3 . E_{1}$ in that figure is the translational energy relative to $v=1$ of $\mathrm{HF}$. We see that both $P_{10}^{R}$ and $P_{10}^{V}$ have very small effective threshold energies $(<0.01 \mathrm{eV})$. Above threshold, the reactive probability is significantly larger than the nonreactive one over much of the energy range scanned. Only in the vicinity of resonances (at $E_{1} \sim 0.3 \mathrm{eV}, 0.9$ $\mathrm{eV}$, and $1.2 \mathrm{eV}$ ) do the two curves cross. In regions where the two curves are smooth (where the direct processes dominate) $P_{10}^{R}$ is usually 5 to 10 times larger than $P_{10}^{V}$. This indicates that reactive collisions are more important than nonreactive ones in producing vibrational deactivation in $\mathrm{H}+\mathrm{FH}(v=1)$ collisions. The same conclusion is usually also true for $\mathrm{H}+\mathrm{FH}(v=2,3)$, as is shown in Figs. 4-8. In Fig. 4 we plot $P_{20}^{R}$ and $P_{20}^{V}$ while $P_{21}^{R}$ and $P_{21}^{V}$ are depicted in Fig. 5 . In both figures, there is essentially zero effective threshold energy for the deactivation processes. Above threshold, we find that $P_{20}^{R}$ is 2 to 20 times larger than $P_{20}^{V} . \quad P_{21}^{R}$ is also significantly larger than $P_{21}^{V}$, but only for the important range of energies below $E_{2}=0.15 \mathrm{eV}$. If the probabilities in Figs. 4 and 5 are compared, we find that the $2 \rightarrow 0$ and $2-1$ transition probabilities are generally comparable in magnitude. This contrasts with the dominance of the $v^{\prime}=v-1$ probability which is of ten obtained (or assumed) in purely nonreactive systems. ${ }^{1}$ As might be expected, the relative strength of the individual $P_{v v^{\prime}}^{V}$ or $P_{v v^{\prime}}^{R}$ for fixed $v$ and varying $v^{\prime}$ is usually dependent on the strength and nature of the interaction potential. For many nonreactive systems, the interaction potential is weak so that all inelastic transition probabilities are small with the $v^{\prime}=v-1$ being the largest (similar to transitions in a perturbed harmonic oscillator). For a reactive surface such as exists for $\mathrm{H}+\mathrm{FH}$ (Fig. 1), severe distortion of the reagent diatomic can occur during the collision so that all inelastic transition probabilities become comparable (and large as well). In Figs. 6-8

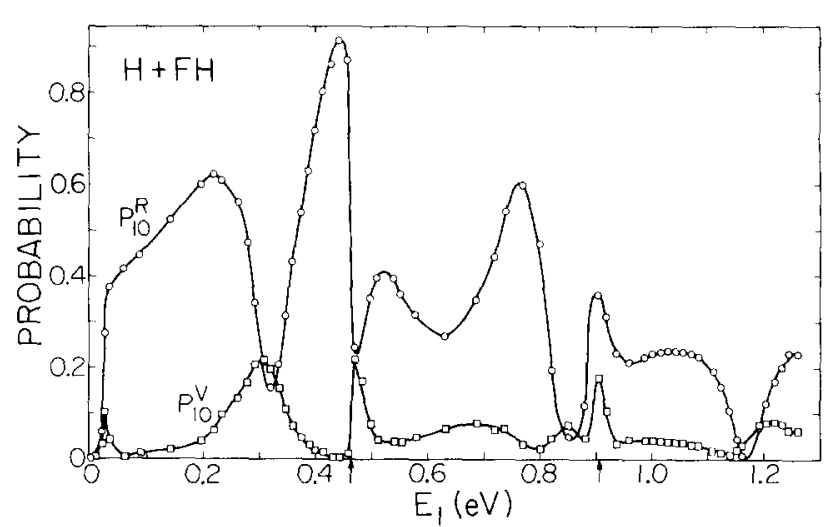

FIG. 3. Transition probabilities $P_{10}^{R}$ (circles) and $P_{10}^{V}$ (squares) for $\mathrm{H}+\mathrm{FH}$ as a function of the reagent relative translational energy $E_{1}$. Arrows in abscissa indicate the energies at which $v=2$ and $v=3$ of $\mathrm{HF}$ open $\left(E_{1}=0.466 \mathrm{eV}\right.$ and $E_{1}=0.909 \mathrm{eV}$, respectively). 


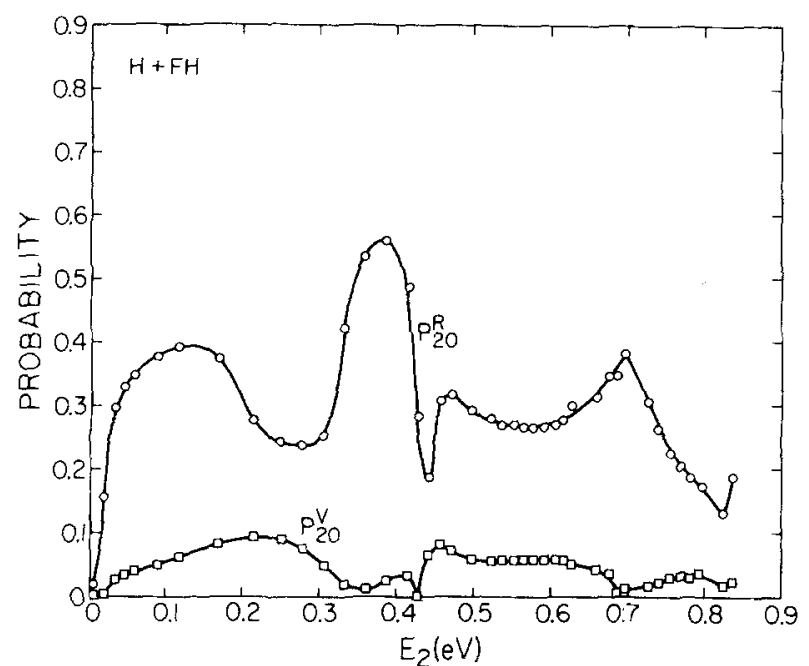

FIG. 4. Transition probabilities $P_{20}^{R}$ (circles) and $P_{20}^{V}$ (squares) for $\mathrm{H}+\mathrm{FH}$ as a function of the reagent relative translational energy $E_{2}$. A rrow at $E_{2}=0.444 \mathrm{eV}$ indicates the energy at which $v=3$ of $H F$ opens.

we plot the inelastic transition probabilities from $v=3$ of $\mathrm{HF}$. Again we see the dominance of the reactive over the nonreactive probabilities (for the same $v \rightarrow v^{\prime}$ process). In addition, the probabilities for multiquantum jump transitions are comparable to (or larger than) those for single jump transitions. Both the preceding conclusions are in agreement with the analogous 3D results of Wilkins. ${ }^{11}$

In Ref. 6 , the collinear $H+H F$ probabilities analogous to those for $\mathrm{H}+\mathrm{FH}$ in Figs. 2-8 were calculated. Over the range of $E_{1}$ from 0.0 to $0.4 \mathrm{eV}$ (important for thermal rate constants) we find that $P_{10}^{V}(\mathrm{H}+\mathrm{HF}) \leq 10^{-2} P_{10}^{V}$ $(\mathrm{H}+\mathrm{FH})$. Evidently, then, the deactivation probabilities are strongly dependent on the orientation of $\mathrm{H}$ with respect to HF. Similar conclusions are valid for the inelastic probabilities from $v=2$ and 3 of HF as well. Also of interest is the fact that for $\mathrm{H}+\mathrm{HF}$ collisions, $P_{21}^{V}$ is usually over 1000 times larger than $P_{20}^{V}$, and $P_{32}^{V}$

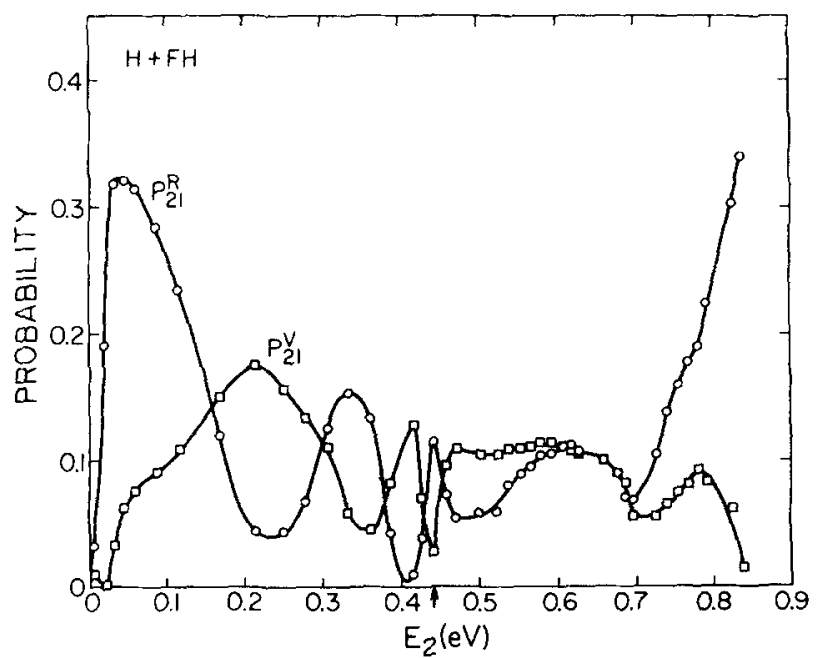

FIG. 5. Transition probabilities $P_{21}^{R}$ (circles) and $P_{21}^{V}$ (squares) for $\mathrm{H}+\mathrm{FH}$ analogous to $\mathrm{Fig}$. 4 .

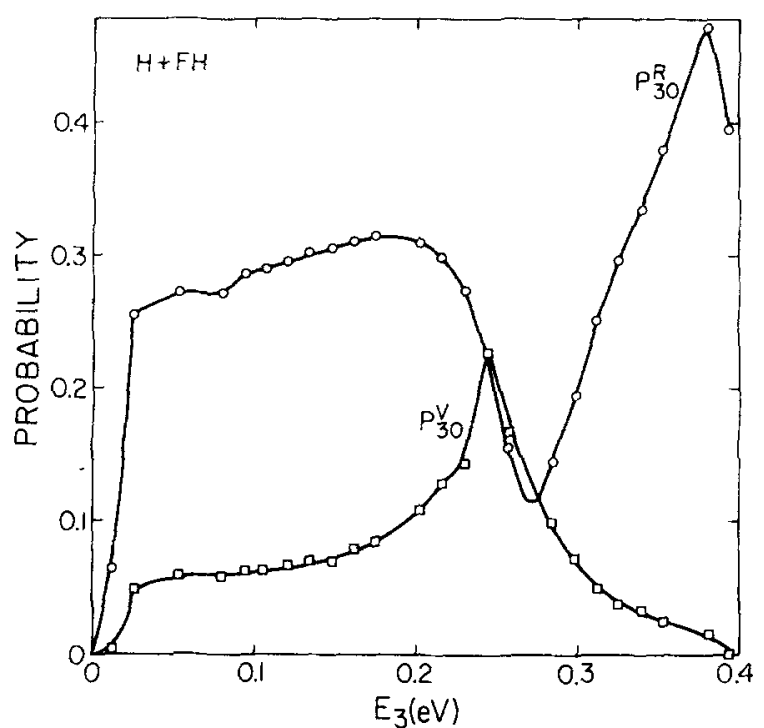

FIG. 6. Transition probabilities $P_{30}^{R}$ (circles) and $P_{30}^{V}$ (squares) for $\mathrm{H}+\mathrm{FH}$ as a function of the reagent relative translational energy $E_{3}$.

$\simeq 100 P_{31}^{V} \simeq 10^{4} P_{30}^{V}$. This indicates the dominance of the $v^{\prime}=v-1$ transition probabilities for $\mathrm{H}+\mathrm{HF}$, in contrast to the behavior of the same probabilities for $\mathrm{H}+\mathrm{FH}$. Such behavior is most easily understood by examining the potential energy surfaces involved. The $\mathrm{H}+\mathrm{HF}$ surface (see Ref. 6) has a largely repulsive nonreactive appearance. [ The barrier for reaction to give $\mathrm{F}+\mathrm{H}_{2}$ is $1.42 \mathrm{eV}(33 \mathrm{kcal} / \mathrm{mole})$.] This implies that the $\mathrm{HF}$ is not significantly stretched or compressed in linear $\mathrm{H}+\mathrm{HF}$ collisions whereas it clearly will be significantly stretched in $\mathrm{H}+\mathrm{FH}$ (Fig. 1) (for the collision energies and initial vibrational states considered), and this difference in the amount of distortion in the HF bond length leads directly to the observed differences in transition probabilities. Notice how this comparison of the extent of bond distortion during collisions is exactly the opposite of what might be expected on the basis of mass effects. As has been pointed out several times, ${ }^{1}$ nonreac-

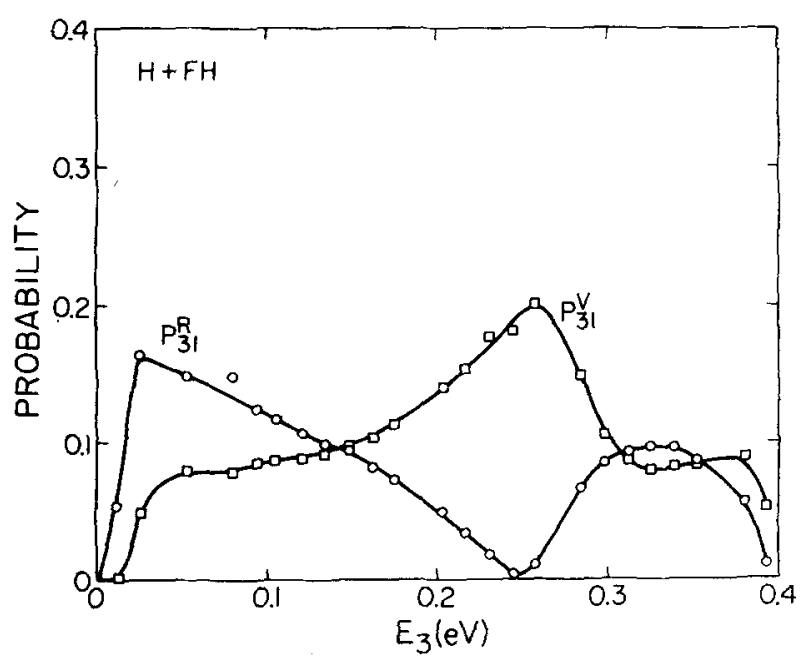

FIG. 7. Transition probabilities $P_{31}^{R}$ (circles) and $P_{31}^{V}$ (squares) for $\mathrm{H}+\mathrm{FH}$ analogous to Fig. 6 . 


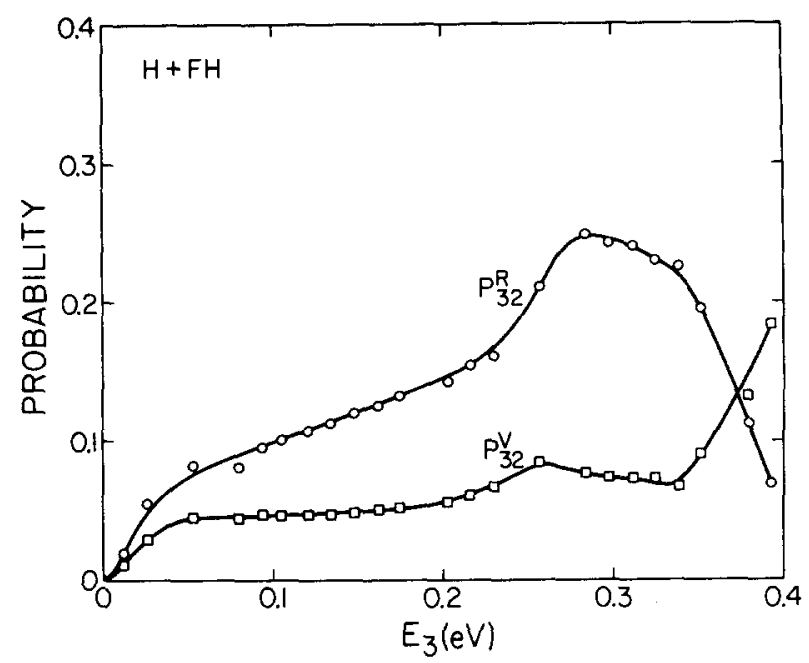

FIG. 8. Transition probabilities $P_{32}^{R}$ (circles) and $P_{31}^{V}$ (squares) for $\mathrm{H}+\mathrm{FH}$ analogous to Fig. 6 .

tive collinear $A+B C$ collision systems having large values of the mass ratio $m=m_{A} m_{C} / m_{B}\left(m_{A}+m_{B}+m_{C}\right.$ ) (as in $\mathrm{H}+\mathrm{HF}$ ) usually show larger bond distortion (and hence larger multiple quantum jump probabilities) than those with smaller $m$ (as $\mathrm{H}+\mathrm{FH}$ ). This assumes that the interaction potentials governing the collisions are all similar and is apparently not the dominant influence in the present case, where the potentials are quite different. We should also remark that the kind of deactivation behavior to be expected in three dimensional collisions will depend on features of the orientation dependence of the interaction potential. For most orientations of $\mathrm{H}$ with respect to HF (roughly $80 \%$ or more), we find potentials similar to the $\mathrm{H}+\mathrm{FH}$ one in Fig. $1 .{ }^{29}$ Only a small range of orientations gives a potential similar to the $\mathrm{H}+\mathrm{FH}$ linear potential, thus indicating that the $\mathrm{H}+\mathrm{HF}$ system should be representative of the majority of collisions. That this conclusion is correct is evident from the qualitative agreement between our $1 \mathrm{D}$ and Wilkins' $3 \mathrm{D}$ results mentioned at the end of the previous paragraph.

We now consider the $v=1$ to $v^{\prime}=0$ inelastic probabilities for $\mathrm{D}+\mathrm{FD}, \mathrm{D}+\mathrm{FH}$, and $\mathrm{H}+\mathrm{FD}$. These are shown in Figs. 9 and 10. In Fig. 10 we have plotted the $\mathrm{D}+\mathrm{FH}$ and $H+F D$ results on an absolute energy scale so as to show the important relationships of the probabilities to one another. Both Figs. 9 and 10 indicate that the reactive probabilities dominate over the nonreactive ones for analogous transitions. However, at low collision energies, $P_{10}^{V}(\mathrm{H}+\mathrm{FD})$ is only slightly smaller than $P_{10}^{R}$ $(\mathrm{H}+\mathrm{FD})$ so the corresponding rate constants should be quite similar in magnitude.

\section{B. Rate constants}

The one dimensional thermal rate constants $k_{v v}$ are obtained from the appropriate Boltzmann average of reagent velocity times transition probability. As shown in Ref. 6, we can write this relationship as

$k_{v v^{\prime}}(T)=(2 \pi \mu k T)^{-1 / 2} \int_{0}^{\infty} P_{v v^{\prime}}\left(E_{v}\right) \exp \left(-E_{v} / k T\right) d E_{v}$,

where $\mu$ is the relative motion reduced mass and $k$ has

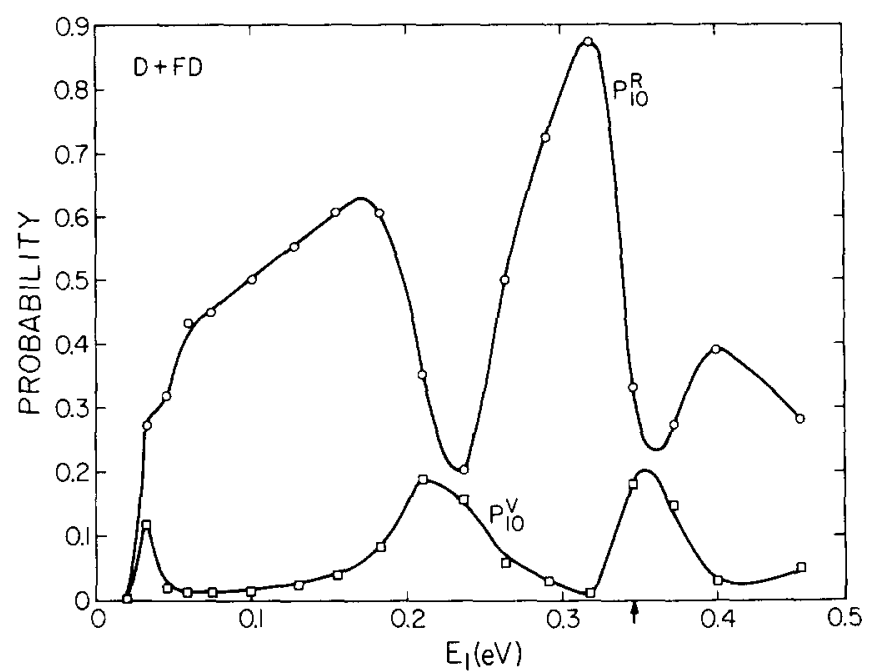

FIG. 9. Transition probabilities $P_{10}^{R}$ (circles) and $P_{10}^{V}$ (squares) for $\mathrm{D}+\mathrm{FD}$ as a function of $E_{1}$. A rrow at $E_{1}=0.345 \mathrm{eV}$ indicates the energy at which $v=2$ of DF opens.

units of $\mathrm{cm} /($ molec $\times \mathrm{sec})$. Note that while a Boltzmann velocity distribution has been assumed, a Boltzmann distribution of vibrational states has not, for we are interested in reagents initially in a single vibrational state $v$.

Using Eq. (B. 1), rate constants for all of the transitions considered in Figs. 3-10 have been calculated. Arrhenius type plots of the $\mathrm{H}+\mathrm{FH}$ rate constants $k_{10}^{R}$, $k_{10}^{V}, k_{20}^{R}, k_{20}^{V}, k_{21}^{R}, k_{21}^{V}, k_{30}^{R}, k_{30}^{V}, k_{31}^{R}, k_{31}^{V}, k_{32}^{R}$, and $k_{32}^{V}$ are given in Figs. 11 and 12 . Note that many of the curves in these two figures are nonlinear. This results from the fact that the corresponding transition probabilities have essentially zero threshold energies and often oscillatory behavior above threshold. As the temperature

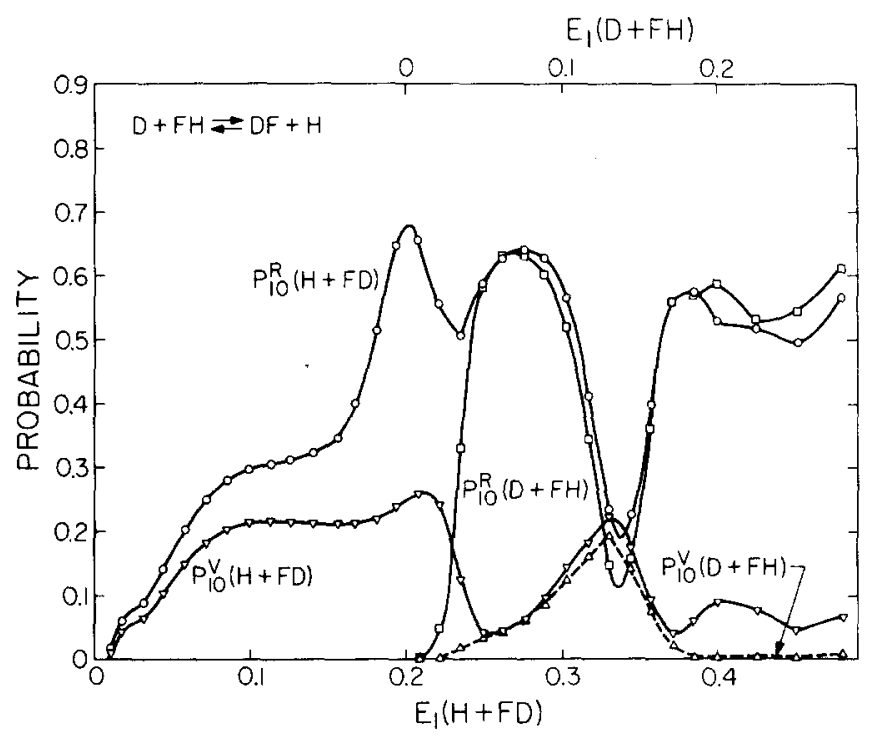

FIG. 10. Transition probabilities $P_{10}^{R}(\mathrm{H}+\mathrm{FD})$ (circles), $P_{10}^{V}$ (H + FD) (triangles), $P_{10}^{R}(\mathrm{D}+\mathrm{FH})$ (squares), and $P_{10}^{V}(\mathrm{D}+\mathrm{FH})$ (triangles and dashed curve) as a function of $E_{1}(\mathrm{H}+\mathrm{FD})$ (lower scale) and $E_{1}(\mathrm{D}+\mathrm{FH})$ (upper scale). The $v=1$ state of $\mathrm{HF}$ opens at $E_{1}(\mathrm{H}+\mathrm{FD})=0.199 \mathrm{eV}$ so the $\mathrm{H}+\mathrm{FD}$ and $\mathrm{D}+\mathrm{HF}$ scales have been displaced by that amount. 


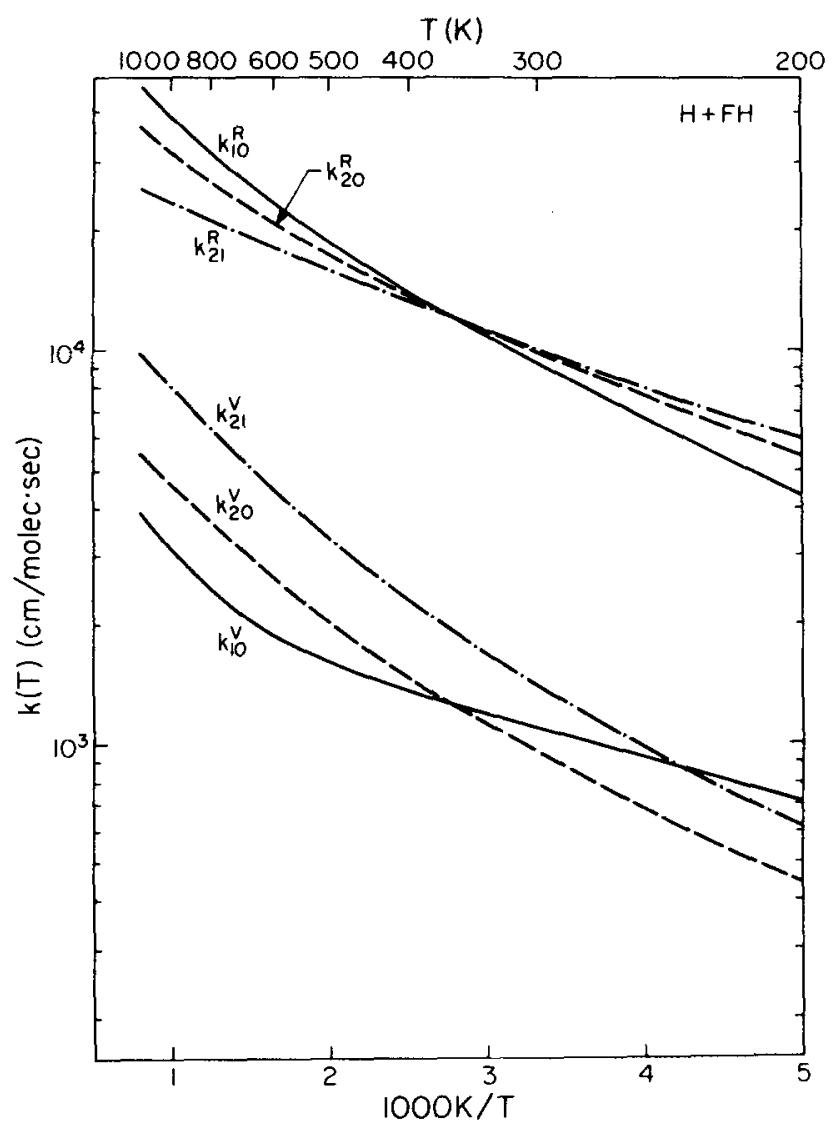

FIG. 11. Arrhenius plot of the rate constants $k_{10}^{R}, k_{10}^{V}$ (solid curves), $k_{20}^{R}, k_{20}^{V}$ (dashed), and $k_{21}^{R}, k_{21}^{V}$ (dash-dotted).

changes, the largest contributions to the integral in Eq. (B. 1) come from gradually changing energies and this results in the changing of the slopes in the Arrhenius plots. To examine the degree of dominance of reactive over nonreactive deactivation mechanisms, let us consider the ratio $R_{v v^{\prime}}=k_{v v^{\prime}}^{R} / k_{v v^{\prime}}^{V}$ at $300{ }^{\circ} \mathrm{K}$. We find that $R_{10}=8.3, R_{20}=10.4, R_{21}=7.1, R_{30}=5.3, R_{31}=2.83$, and $R_{32}=1.89$. Note that the reactive and nonreactive rate constants approach each other as $v$ approaches $v^{\prime}$. In contrast, the ratio $k_{v}^{R} / k_{v}^{V}$ of total inelastic rate constants $k_{v}^{R}$ and $k_{v}^{V}$, (obtained by summing $k_{v v}^{R}$ and $k_{v v^{\prime}}^{V}$ over all $v^{\prime}$ $\neq v$ ) has the value 8.3 for $v=1,8.5$ for $v=2$, and 3.5 for $v=3$ and thus shows no systematic variation with increasing $v$. The total deactivation rate constant $k_{v}=k_{v}^{R}$ $+k_{v}^{V}$ depends on $v$ in a way which reflects the differences in the total inelastic transition probabilities (obtained by summing the probabilities in Figs. $3-8$ ). The ratio $k_{2} / k_{1}$, for example, has the value 2.1 at $300^{\circ} \mathrm{K}$ while $k_{3} / k_{2}=0.94$ at the same temperature. In his classical trajectory study ${ }^{11}$ Wilkins found $k_{2} / k_{1} \simeq 3.9$ and $k_{3} / k_{2}$ $\simeq 1$. 8. Neither of these results is consistent with the rule $k_{v, v-1}=v k_{10}$.

Considering now the rate constants for $\mathrm{H}+\mathrm{FH}, \mathrm{D}+\mathrm{FD}$, $\mathrm{H}+\mathrm{FD}$, and $\mathrm{D}+\mathrm{FH}$, we find that the ratio $R_{10}=k_{10}^{R} / k_{10}^{V}$, has the value 8.3 for $\mathrm{H}+\mathrm{FH}$ and $\mathrm{D}+\mathrm{FD}, 1.4$ for $\mathrm{H}+\mathrm{FD}$, and 12.0 for $\mathrm{D}+\mathrm{FH}$ (all at $300^{\circ} \mathrm{K}$ ). The low value for $\mathrm{H}+\mathrm{FD}$ is clearly a consequence of the great similarity of $P_{10}^{R}(\mathrm{H}+\mathrm{FD})$ and $P_{10}^{V}(\mathrm{H}+\mathrm{FD})$ in Fig. 10. The corresponding ratios $R_{10}$ calculated from Wilkins' results (see Ref. 4) are 3.2 for $\mathrm{H}+\mathrm{FH}, 9.0$ for $\mathrm{D}+\mathrm{FD}, 1.3$ for $\mathrm{H}+\mathrm{FD}$, and 9.0 for $\mathrm{D}+\mathrm{HF}$. These numbers are in reasonable agreement with ours although we should emphasize that the validity of this comparison is questionable without some consideration of the effect of rotations (such as has been discussed by Levine and Bernstein ${ }^{30}$ ).

\section{SUMMARY}

We now summarize the important results of this paper. First, for all transition probabilities and rate constants in the four collision systems (R. 1-R.5), the reactive mechanism dominates over the nonreactive one in producing vibrational deactivation. This result is apparently of general validity over a wide range of impact parameters and diatomic orientations since the same conclusions were obtained by Wilkins in his 3D classical calculations. Second, multiquantum jump transition probabilities are comparable in magnitude to single quantum jump transition probabilities. This is clearly a consequence of the use of a low barrier reactive potential energy surface since the analogous $\mathrm{H}+\mathrm{HF}$ results (for a high barrier surface) indicate that single quantum jump transition probabilities are orders of magnitude larger than all others at the energies considered. Third, an examination of vibrational deactivation as a function of isotopic composition indicates strong sensitivity of the ratio of reactive to nonreactive rates to this composition. The comparison of $k_{10}^{R} / k_{10}^{V}$ for $\mathrm{H}+\mathrm{FD}$ vs D $+\mathrm{FH}$ $(1.4$ vs 12.0$)$ is especially interesting since the prod-

$T(K)$

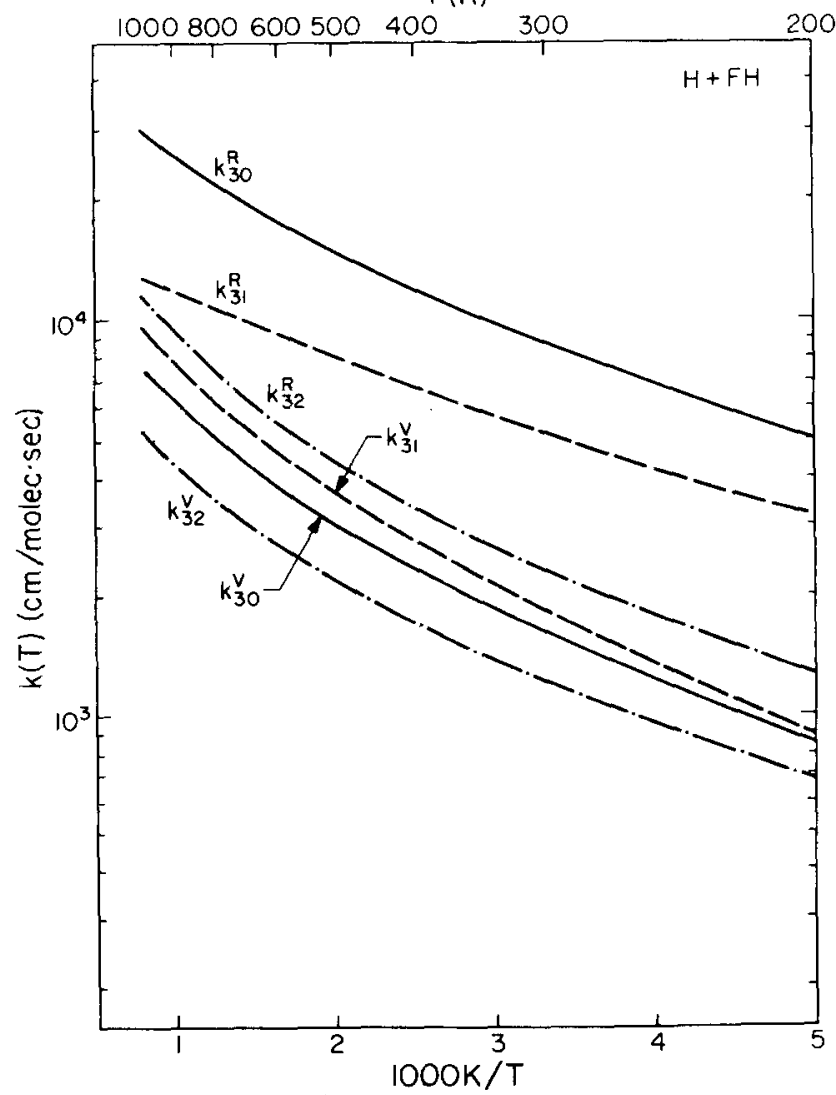

FIG. 12. Arrhenius plot of the rate constants $k_{30}^{R}, k_{30}^{V}$ (solid curves), $k_{31}^{R}, k_{31}^{V}$ (dash), and $k_{32}^{R}, k_{32}^{V}$ (dash-dotted). 
ucts of both deactivation pathways are distinguishable, which means that these ratios can be measured experimentally. In addition, we expect these ratios of reactive to nonreactive rates to be very sensitive to barrier height.

Finally, we should again state that although the potential surface used in this study is of questionable validity, there are a number of similar chemically reactive systems which could be important in vibrational deactivation to which the surface might be applicable, and present results should be a useful guide to understanding them.

${ }^{1}$ For reviews of nonreactive vibrational deactivation processes, see D. Rapp and T. Kassal, Chem. Rev. 69, 61 (1969); and J. P. Toennies, Annu. Rev. Phys. Chem. 26, 259 (1975).

${ }^{2}$ (a) G. P. Quigley and G. J. Wolga, Chem. Phys. Lett. 27, 276 (1974); J. Chem. Phys. 63, 5263 (1975); (b) R. L. Wilkins, J. Chem. Phys. 59, 698 (1973).

${ }^{3} \mathrm{M}$. A. Kwok and R. L. Wilkins, J. Chem. Phys. 60, 2189 (1974).

${ }^{4}$ R. F. Heidner and J. F. Bott, J. Chem. Phys. 63, 1810 (1975); J. F. Bott, J. Chem. Phys. 65, 1976 (1976).

${ }^{5}$ (a) A. Kuppermann, Potential Energy Surfaces in Chemistry, edited by W. A. Lester (University of California, Santa Cruz, 1970), p. 121-129; (b) Abstracts of papers, VII International Conference on the Physics of Electronic and Atomic Collisions, Amsterdam, 1971.

${ }^{6}$ G. C. Schatz, J. M. Bowman, and A. Kuppermann, J. Chem. Phys. 63, 674 (1975).

${ }^{7}$ G. C. Schatz, J. M. Bowman, and A. Kuppermann, J. Chem. Phys. 63, 685 (1975).

${ }^{8}$ G. C. Schatz and A. Kuppermann, J. Chem. Phys. 65, 4624, 2688 (1976)

${ }^{9}$ G. C. Schatz and A. Kuppermann, Phys. Rev. Lett. 35, 1266 (1975).

${ }^{10}$ M. Baer, J. Chem. Phys. 65, 493 (1976).

${ }^{11}$ R. L. Wilkins, J. Chem. Phys. 58, 3038 (1973); Mol. Phys. 29, 555 (1975).

${ }^{12}$ D. L. Thompson, J. Chem. Phys. 57, 4170 (1972).
${ }^{13}$ D. L. Thompson, J. Chem. Phys. 57, 4164 (1972).

${ }^{14} \mathrm{~S}$. V. O'Neill, H. F. Schaefer, and C. F. Bender, Proc. Nat. A cad. Sci. USA 71, 104 (1974).

${ }^{15}$ C. A. Parr and D. G. Truhlar, J. Phys. Chem. 75, 1844 (1971).

${ }^{16}$ C. F. Bender, B. J. Garrison, and H. F. Schaefer, J. Chem. Phys. 62, 1188 (1975); P. Botschwina and W. Meyer, Chem. Phys. 20, 43 (1977); W. R. Wadt and N. Winter, J. Chem. Phys. 67, 3069 (1977).

${ }^{17}$ F. E. Bartaszek, D. M. Manos, and J. C. Polanyi, J. Ċhem. Phys. 69, 933 (1978).

${ }^{18} \mathrm{~J}$. P. Dwyer and A ron Kuppermann, to be published; see also J. P. Dwyer, Ph. D. Thesis, California Institute of Technology, 1977.

${ }^{19}$ G. C. Schatz and A. Kuppermann, J. Chem. Phys. 59, 964 (1973).

${ }^{20}$ G. C. Schatz, J. M. Bowman, and A. Kuppermann, J. Chem. Phys. 58, 4023 (1973).

${ }^{21}$ (a) J. T. Muckerman, J. Chem. Phys. 54, 1155 (1971); (b) ibid. 56, 2997 (1972).

${ }^{22} \mathrm{~J}$. T. Muckerman, private communication.

${ }^{23}$ The value of $B_{\rho}(\mathrm{HF})$ used in these calculations was $2.2087 \AA^{-1}$ (not 2.2187 $\breve{A}^{-1}$ as was used in Ref. 8). The parameter $R_{e}(\mathrm{~Hz})$ used in both the present calculations and those of Ref. 8 has the value $0.7419 \AA$ (not $0.749 \AA$ as stated in Ref. 8).

${ }^{24}$ Since the product of the $\mathrm{H}+\mathrm{FD}$ reaction is $\mathrm{D}+\mathrm{FH}$ and vice versa, symmetry of the scattering matrix requires that $P_{00}^{R}$ $(\mathrm{H}+\mathrm{FD})$ equal $P_{00}^{R}(\mathrm{D}+\mathrm{FH})$ when both are evaluated at the same total energy. When plotted as a function of their respective reagent translational energies, these two identical probabilities will be displaced by the (negative of the) difference in vibrational energies $(0.069 \mathrm{eV}$ in this case).

${ }^{25}$ D. G. Truhlar and A. Kuppermann, J. Chem. Phys. 56, 2232 (1972).

${ }^{26}$ D. J. Diestler, J. Chem. Phys. 54, 4547 (1971).

${ }^{27}$ D. G. Truhlar, A. Kuppermann, and J. T. Adams, J. Chem. Phys. 59, 395 (1973).

${ }^{28}$ G. C. Schatz and A ron Kuppermann, to be published; see also G. C. Schatz, Ph. D. thesis, California Institute of Technology, 1975.

${ }^{29}$ G. C. Schatz and A. Kuppermann (unpublished results).

${ }^{30}$ R. D. Levine and R. B. Bernstein, Chem. Phys. Lett. 29, 314 (1974). 